\title{
PRÁTICA EDUCATIVA DE ENFERMEIRAS NA ATENÇÃO PRIMÁRIA À SAÚDE, PARA O DESENVOLVIMENTO INFANTIL SAUDÁVEL
}

Mayara de Melo Pereira' ${ }^{1}$, Thiffany Pestana da Penha², Daniele de Souza Vieira ${ }^{3}$, Elenice Maria Cecchetti Vaz ${ }^{4}$, Nathaniely Cristina Carvalho de Brito Santos ${ }^{5}$, Altamira Pereira da Silva Reichert ${ }^{6}$

${ }^{1}$ Enfermeira. Estratégia de Saúde da Família da Prefeitura Municipal de Icó. Icó, CE, Brasil.

${ }^{2}$ Enfermeira. Maternidade Frei Damião. João Pessoa, PB, Brasil.

${ }^{3}$ Enfermeira. Mestranda em Enfermagem. Universidade Federal da Paraíba. João Pessoa, PB, Brasil.

${ }^{4}$ Enfermeira. Doutoranda em Enfermagem. Docente do Departamento de Enfermagem Materno-Infantil e Psiquiátrica da Universidade Federal Fluminense. Niterói, RJ, Brasil.

${ }^{5}$ Enfermeira. Doutoranda em Enfermagem. Docente de Enfermagem da Universidade Federal de Campina Grande. Cuité, PB, Brasil.

${ }^{6}$ Enfermeira. Doutora em Saúde da Criança e do Adolescente. Docente do Departamento de Enfermagem de Saúde Pública e Psiquiatria do Centro de Ciências da Saúde e do Programa de Pós-Graduação em Enfermagem da Universidade Federal da Paraíba. João Pessoa, PB, Brasil.

RESUMO: O objetivo desse estudo foi identificar a concepção de educação em saúde que norteia a prática educativa de enfermeiras que atuam na Atenção Primária à Saúde, visando à promoção do desenvolvimento infantil saudável. Os dados foram coletados de setembro de 2012 a janeiro de 2013, por meio de entrevista realizada com 10 enfermeiras que atuam na Estratégia Saúde da Família no município de João Pessoa-Paraíba. O material empírico foi tratado a partir de análise categorial temática. Percebeu-se que há enfermeiras que desconhecem a essência do processo de educação em saúde, mantendo concepções biologicistas, enquanto outras referem desenvolver prática educativa somente durante a consulta de puericultura, porém, não contempla a estimulação do desenvolvimento neuropsicomotor. Diante disso, surge a necessidade de despertar o pensar/ agir dessas enfermeiras, a fim de ampliar o conceito de educação em saúde e implementá-las na vigilância do desenvolvimento infantil para transformar suas práticas.

DESCRITORES: Educação em saúde; Atenção primária à saúde; Desenvolvimento infantil; Enfermagem pediátrica.

\section{NURSING EDUCATIONAL PRACTICE IN PRIMARY HEALTH CARE AIMED TO HEALTHY CHILD DEVELOPMENT}

ABSTRACT: The purpose of the present study is to identify the concept of health education that guides the educational practice of nurses who work in Primary Health Care, aimed at promoting children's healthy development. The data were collected from September 2012 to January 2013, through interviews with 10 nurses who work in the Family Health Strategy, in the city of João Pessoa-Paraíba. Thematic analysis was the technique used in the treatment of the empirical material. Some nurses were unaware of the importance of the health education process, keeping biologicist views of this process, while others reported developing educational practices only during child care consultation, which however did not include stimulation of neuropsychomotor development. Therefore, it is necessary to awaken the thinking/acting of these nurses, in order to broaden the concept of health education for including child development monitoring in their practices.

DESCRIPTORS: Health education: Primary health care; Child development; Pediatric nursing.

\section{PRÁCTICA EDUCATIVA DE ENFERMERAS EN LA ATENCIÓN PRIMARIA A LA SALUD, PARA EL DESARROLLO INFANTIL SALUDABLE}

RESUMEN: El objetivo de ese estudio fue identificar la concepción de educación en salud que nortea la práctica educativa de enfermeras que trabajan con Atención Primaria a la Salud, para la promoción del desarrollo infantil saludable. Los datos fueron obtenidos de septiembre de 2012 a enero de 2013, por medio de entrevista realizada con 10 enfermeras que actuan en la Estrategia Salud de la Familia en municipio de João Pessoa, Paraíba. El material empírico fue tratado con base en el análisis categorial temático. Se percibió que hay enfermeras que desconocen la esencia del proceso de educación en salud, manteniendo concepciones biologicistas, en cuanto otras afirman desarrollar práctica educativa solamente durante la consulta de puericultura, pero no contempla el estímulo del desarrollo neuropsicomotor. Delante de eso, surge la necesidad de despertar el pensar/ actuar de esas enfermeras, para ampliar el concepto de educación en salud y implementarlas en la vigilancia del desarrollo infantil para cambiar sus prácticas.

DESCRIPTORES: Educación en salud; Atención primaria a la salud; Desarrollo infantil; Enfermería pediátrica. 


\section{INTRODUÇÃO}

O desenvolvimento infantil constitui-se em um processo resultante da interação entre os fenômenos de crescimento, maturação e aprendizagem, cujas funções são identificadas em habilidades e comportamentos nas dimensões física, intelectual, emocional e social ${ }^{(1)}$.

Os primeiros anos de vidas são um período crítico na vida da criança, tendo em vista o intenso desenvolvimento cerebral, que a torna mais suscetível a influências positivas ou negativas das experiências. Diante disto, vê-se a relevância da efetivação de ações de educação em saúde, por considerá-las ideias para a promoção do desenvolvimento infantil saudável ${ }^{(2)}$.

A educação em saúde representa um dos principais elementos para a promoção do desenvolvimento saudável, pois possibilita ao cuidador da criança a formação de uma consciência crítica e reflexiva, por meio da produção de um saber para autonomia no cuidar não só de si, mas de toda a família, com enfoque especial à criança ${ }^{(3)}$.

$\mathrm{Na}$ atenção à criança, a promoção da saúde apresenta uma estreita relação com a vigilância em saúde e desenvolvimento infantil, cujas ações são capazes de reduzir situações de vulnerabilidade e riscos, favorecendo a identificação e a intervenção precoce frente ao atraso no desenvolvimento infantil( $^{(4)}$.

Contudo, a carência de ambientes adequados e deficitárias atividades educativas voltadas para a promoção do cuidado infantil, por vezes, dificultam a relação e o diálogo franco entre cuidador/mãe/profissional de saúde, com consequente falta de esclarecimento de possíveis dúvidas durante o processo de cuidar da criança enquanto ser integral(5).

Assim, a educação em saúde, considerada uma das ferramentas indispensáveis ao trabalho do profissional de saúde, deve enfocar o cuidado às crianças, pois estas, quando acompanhadas adequadamente, possivelmente adquirem a capacidade de na idade adulta tornarem-se pessoas aptas a refletir sobre seu contexto e seu papel enquanto cidadão comprometido com sua qualidade de vida e saúde ${ }^{(6)}$.

Nesse contexto, torna-se enfático destacar a educação em saúde entre as atribuições do enfermeiro na vigilância do desenvolvimento da criança, especialmente nos serviços de Atenção Primária à Saúde (APS), ao viabilizar a identificação de possíveis alterações no crescimento e desenvolvimento e possibilitar intervenções oportunas, a fim de favorecer melhor qualidade de vida à população infantil(7).

Diante dessas reflexões, o estudo justificase por mostrar a importância das atividades educativas de enfermeiras para a promoção do desenvolvimento infantil saudável, contribuindo para a qualidade do cuidado, principalmente nos dois primeiros anos de vida, visto ser o período fundamental para o acompanhamento das crianças a fim de monitorá-las e promover o seu potencial, correspondente à faixa etária. Neste sentido, a Estratégia Saúde da Família (ESF) torna-se grande aliada para a efetividade desse processo, tendo em vista seu foco na prevenção e promoção da saúde, a partir de uma visão ampliada do indivíduo, do respeito e valorização da família, e utilização de práticas de educação em saúde.

Dada a relevância da temática e da observação de fragilidades em algumas práticas educativas de enfermeiras que prestam assistência à criança, percebeu-se a necessidade de refletir sobre tais práticas, a fim de contribuir para promover o acompanhamento adequado e eficaz do desenvolvimento infantil. A partir do resultado dessa pesquisa também será possível refletir sobre a realidade exercida por esses profissionais na atenção à criança.

Portanto, o objetivo deste estudo consiste em identificar a concepção de educação em saúde que norteia a prática educativa de enfermeiras da Atenção Primária à Saúde, visando à promoção do desenvolvimento infantil saudável.

\section{METODOLOGIA}

Trata-se de pesquisa exploratório-descritiva e de campo, com abordagem qualitativa, realizada entre setembro de 2012 e janeiro de 2013, em Unidades Integradas de Saúde da Família pertencentes ao Distrito Sanitário III da cidade de João Pessoa, estado da Paraíba. Optou-se pela Unidade Integrada pela facilidade de acesso aos profissionais que lá trabalham, tendo em vista que em cada uma destas atuam quatro equipes completas de Saúde da Família.

Os participantes da pesquisa foram 10 enfermeiras, funcionárias das referidas unidades de saúde e que atendem às famílias de crianças nelas cadastradas. Foram excluídos do estudo, enfermeiros que estavam de férias ou licenciados no período da coleta de dados. O encerramento 
da coleta seguiu o critério de suficiência, ou seja, quando o julgamento de que o material empírico permite traçar um quadro compreensivo do objeto de estudo. Para garantia do anonimato, os sujeitos participantes foram identificados com a letra $E$, correspondente à categoria enfermeiro, seguido do número arábico da sequência da entrevista.

Para coleta de dados empíricos utilizou-se entrevista semiestruturada, a partir das questões norteadoras: $\mathrm{O}$ que você entende por Educação em Saúde? Você realiza alguma prática educativa junto à família de crianças menores de dois anos, para a promoção do desenvolvimento saudável? Quais as ações educativas que você desenvolve?

As entrevistas foram previamente agendadas conforme conveniência das participantes, com exceção daquelas que preferiram realizá-la logo no primeiro contato com a entrevistadora, nas próprias Unidades. As falas foram gravadas em mídia digital, após anuência das entrevistadas por meio da assinatura do Termo de Consentimento Livre e Esclarecido, garantindo-Ihes o anonimato e sigilo de informações. Posteriormente, foram transcritas na íntegra, a fim de captar informações indispensáveis à realização do estudo.

A interpretação dos dados seguiu os passos da análise categorial temática(8), na qual se deu primeiramente a organização do material coletado em determinada ordem, já iniciando uma classificação. Após isso, traçou-se o mapa horizontal do material e, posteriormente, à luz dos objetivos propostos, realizou-se sucessivas leituras dos textos, fazendo uma relação interrogativa para apreensão das estruturas de relevância. Elaborou-se uma classificação por meio da leitura transversal que, a partir das estruturas de relevância, processou-se síntese da classificação, reagrupando os temas mais relevantes para a análise final.

A pesquisa foi aprovada pelo Comitê de Ética do Centro de Ciências da Saúde da Universidade Federal da Paraíba, sob o protocolo ${ }^{\circ}$ 0096/12, CAAE: 02584212.3.0000.5188.

\section{RESULTADOS}

As 10 enfermeiras tinham tempo de formação variando entre 10 e 30 anos e, em sua maioria, possuíam mais de uma especialização; sete possuíam especialização em Saúde da Família, duas em Saúde Coletiva, duas em Saúde Pública, uma em Auditoria, uma em Unidade de Terapia
Intensiva (UTI); uma possuía Residência em Saúde da Família, e apenas uma não possuía especialização.

A partir da análise dos dados foram construídas duas categorias empíricas: Educação em saúde como ferramenta de promoção da saúde; e Práticas educativas desenvolvidas junto às famílias de crianças menores de dois anos.

\section{Educação em saúde como ferramenta de promoção da saúde}

A partir da análise dos depoimentos, foi possível explanar a compreensão das participantes do estudo acerca da educação em saúde nas suas atividades diárias. Nesse sentido, algumas enfermeiras apresentam uma concepção de educação em saúde como um novo modo de fazer em direção à consolidação dos princípios do Sistema Único de Saúde, compreendendo-a como forma de promover saúde, seja individualmente ou no coletivo.

Educação em saúde é uma forma de promover saúde também, é o primeiro e essencial passo, a educação está ligada à saúde sempre, não é? A partir dos conceitos básicos a gente tem saúde. E educação em saúde no meu serviço é promover saúde através de conversas, de atividades educativas mesmo, na comunidade, dentro do consultório, fora do consultório, na área, como a gente chama no território, é isso! Educação em saúde é promover saúde. (E6)

Educação em saúde a gente faz todos os dias aqui, não é? Não só as palestras que a gente faz com um grupo específico, um grupo prioritário, que a gente tenha [...] nas nossas consultas de enfermagem, durante nossas visitas domiciliares, onde a gente faz nossas orientações, não é? Para promover o bemestar do coletivo, no caso. (E7)

Bom, a gente enfermeira é constante educador, a gente não consegue fazer saúde, sem fazer educação junto. Constantemente, em consultas, em qualquer ação, o papel da gente é educar, acima de levar em consideração essa parte curativa, eu acho que a parte preventiva, essa parte preventiva passa pela educação. (E8)

Educação em Saúde é um conjunto de ações que visa à prevenção de doenças, de levar os usuários a entender o seu processo de saúde-doença, de entender que eles têm participação na sua saúde, seus direitos, ou seja, é um conjunto que integra todos os princípios do SUS, educar o usuário para que ele tenha direito à saúde dele. (E3) 
São orientações que você pode fornecer ao usuário quanto a como ele deve viver melhor, o que ele pode fazer para viver bem e afastando-se das doenças ou convivendo com elas de uma forma menos complicada. (E10)

[Educação em saúde] São ações desenvolvidas com grupos específicos e/ou comunidade, para prevenção e promoção a saúde. (E9)

Verifica-se que as enfermeiras entrevistadas compreendem a educação em saúde como uma prática intimamente ligada à promoção da saúde. A partir disso, a enfermeira atuando como educador, fundamenta-se na prerrogativa de desenvolver ações voltadas para a prevenção e promoção da saúde.

Apesar de uma enfermeira ter expressado a compreensão de que a educação em saúde visa à prevenção e promoção da saúde, seu discurso ainda contém resquícios de uma relação com o usuário baseada na transmissão de informação prescritiva, pois visualiza as suas ações educativas somente com grupos específicos, não vislumbrando que o encontro com o outro no cuidado é permeado pela educação para a saúde do indivíduo e/ou coletividade.

Vale ressaltar que algumas profissionais ainda mantêm a concepção higienista de educação em saúde, ou seja, com atividades voltadas apenas para a prevenção de doenças, conforme depoimentos abaixo. Destaca-se ainda que algumas enfermeiras ressaltam a importância do processo de educação em saúde para a promoção da qualidade de vida do indivíduo, salientando a responsabilidade de toda a equipe de saúde em promover o bem-estar ao usuário.

Educação em Saúde seriam ações, não é? Para que, a gente procura desenvolver com os usuários, não é? Para que a gente possa estar prevenindo doenças. (E2)

É o processo onde os profissionais de saúde, onde a equipe de saúde deve informar aos usuários sobre práticas que venha a proporcionar aos usuários mais saúde, uma qualidade de vida. (E1)

\section{Práticas educativas na Atenção Primária à Saúde da criança desenvolvida junto às famílias}

Nos depoimentos a seguir, as enfermeiras afirmam orientar a mãe/responsável pela criança acerca de assuntos voltados para questões higienistas, vacinal e nutricional durante atividades educativas individuais (nos consultórios) e coletivas (salas de espera, rodas de conversa, palestras). No entanto, não remetem à vigilância do crescimento e desenvolvimento infantil entre suas ações educativas rotineiras, o que mostra a fragilidade dessas ações quando não abordadas nas consultas de puericultura ou outro tipo de atendimento.

Sim, durante a puericultura, não é? $O$ acompanhamento do crescimento e desenvolvimento da criança, a gente trabalha isso com as genitoras, faz as orientações em relação à higiene e profilaxia, a questão da imunização, atualização do cartão vacinal, alimentação saudável, não é? Mas essa educação em saúde da gente é mais dentro do consultório, a gente não tem um grupo específico não. Assim, para gente reunir essas mães e orientar tudinho, não. (E7)

Sim, nós temos várias atividades. Nessa faixa etária, principalmente, fazendo rodas de conversas com as mães sobre imunização, sobre alimentação saudável, sobre cuidados de higiene, tem vários temas para estar abordando, principalmente conversando com as mães, não é? Com as crianças nós também temos atividades, tipo teatrinho com os fantoches, é... dramatizações, apresentações de vídeo, realizadas em creche. (E1)

Sim, nós fazemos. Nós temos o pessoal da puericultura, e nós procuramos fazer sala de espera com eles. Fazemos também palestras com eles em berçários, aí assim os temas são variados, desde alimentação mais saudável, a questão da importância das vacinas, não é? A questão de higiene, a questão do cuidado com ela [criança], quem cuida, como cuida. (E2)

A partir dos depoimentos, foi observado que algumas enfermeiras realizam atividades educativas em vários cenários, e aproveitam o momento da sala de espera para fazer rodas de conversas e orientações à mãe/cuidadora, porém, sem fazer menção à promoção do desenvolvimento saudável.

Salienta-se que uma enfermeira refere dificuldades em realizar atividades educativas de promoção da saúde infantil devido às inúmeras atribuições da enfermeira atuante na atenção básica.

Hoje, a gente faz mais no consultório mesmo. A demanda termina tomando muito da gente, e acabava ficando muito no consultório, por isso, a gente teve essa iniciativa de fazer a puericultura coletiva e para os alunos [da Universidade] fazerem também com a gente. Então faz atividades educativas na puericultura, junto com a médica e os outros profissionais da equipe, mas também não deixa 
de fazer no consultório, a gente orienta a mãe, faz aquela consulta individual, em particular. (E6)

\section{DISCUSSÃO}

Com a implantação da ESF, em 1994, uma nova conjuntura passou a fazer parte dos serviços de saúde brasileiros, com ações de prevenção de agravos e promoção da saúde, entre os quais se destaca a educação em saúde, visto a capacidade de transformar o modo de viver das pessoas ${ }^{(9)}$.

Na mudança de modelo de atenção à saúde, a educação passou a apresentar-se como ferramenta relevante na promoção da saúde, por meio de ações educativas e preventivas, rumo à efetivação do cuidado integral e humanizado ${ }^{(10)}$.

Esse fato é corroborado por estudo(11) realizado, o qual constatou que, para a maioria dos profissionais de saúde atuantes e gestores municipais da ESF, promoção da saúde constituise como práticas assistenciais, com orientações individuais e familiares que são realizadas durante as consultas, nas visitas domiciliares e em grupo, estando assim intimamente ligada à educação em saúde.

A educação em saúde se apresenta como uma estratégia que visa capacitar o indivíduo a uma melhor qualidade de vida, em um processo que envolve a participação de toda a população no contexto do seu cotidiano, levando-o a refletir sobre sua condição no ambiente em que vive, assim contribuindo para atitudes favoráveis ao cuidado em saúde ${ }^{(12)}$. Dessa forma, quando há promoção da saúde, é possível fornecer aos usuários a autonomia no cuidado e empoderamento do processo saúde-doença, a fim de promover mudanças efetivas na vida do sujeito $^{(13)}$.

É preciso ressaltar o discurso de uma enfermeira, que remete a educação em saúde somente a grupos específicos, o que torna notória a necessidade de ampliar a visão acerca dessa ferramenta, pois esta prática pode ser realizada em qualquer ciclo de vida do indivíduo, como em todo contato entre profissional e usuário.

No discurso de algumas profissionais, mantêmse a concepção de educação em saúde pautada no modelo biomédico, valorizando apenas atividades de prevenção a doenças. Coaduna com este achado estudo ${ }^{(14)}$ que objetivou compreender a educação em saúde no contexto da ESF, a partir da literatura brasileira sobre o assunto e com enfoque na atuação da enfermagem. Os resultados evidenciaram que o discurso do enfermeiro detém resquícios do modelo sanitário brasileiro prescritivo, destacando a vigilância para as doenças e a capacidade para intervir e modificar comportamentos da população considerados prejudiciais à saúde.

Essa visão pode ser complementada por outras evidências científicas ${ }^{(15,11)}$ que destacaram a dificuldade que os profissionais de saúde apresentam para diferenciar promoção da saúde e prevenção de doenças, haja vista que as tratam como sinônimo e, por isso, as ações estavam voltadas para a prevenção de doença e de risco.

Talvez essa realidade esteja existindo, porque mesmo a enfermeira se reconhecendo como educadora, as práticas educativas tendem a ser vistas como uma ação técnica, adicional ao conjunto de práticas profissionais, em lugar de uma dimensão inerente e essencial à prática profissional. Por vezes, esta dimensão é tida como mais uma responsabilidade ou tarefa a ser incluída no processo de trabalho, e acaba por reproduzir a racionalidade biomédica hegemônica ${ }^{(16)}$.

No tocante ao cuidado às crianças, é de extrema importância que a educação em saúde seja efetivada, visando proporcionar melhoria na qualidade devida e promoção de um crescimento e desenvolvimento saudável. Algumas enfermeiras relataram realizar ações de educação em saúde a partir de orientações acerca da promoção da saúde das crianças, as quais acontecem durante os momentos para o acompanhamento do crescimento e desenvolvimento infantil, tanto em momentos individuais durante os atendimentos, como nos espaços coletivos que antecedem as consultas.

$\mathrm{Na}$ perspectiva do desenvolvimento infantil e das práticas educativas desenvolvidas junto às famílias, salienta-se que apenas uma enfermeira referiu orientar as mães quanto à estimulação do desenvolvimento neuropsicomotor, estando os demais discursos atrelados somente a orientações pertinentes aos cuidados gerais na infância.

Em relação ao monitoramento e estimulação do desenvolvimento neuropsicomotor às crianças, percebe-se nesta pesquisa a ausência de ações voltadas à vigilância do desenvolvimento infantil. Esse fato corrobora com a pesquisa ${ }^{(17)}$ que avaliou as dificuldades e facilidades enfrentadas no processo de implementação dos conhecimentos adquiridos em um programa de capacitação em vigilância do desenvolvimento infantil, dirigido a enfermeiras da ESF, que constatou que a 
maioria das participantes do estudo não realizava o acompanhamento do desenvolvimento das crianças e as que realizavam demonstravam despreparo na sua execução.

Esse aspecto é preocupante e evidencia uma lacuna na formação dos enfermeiros, pois os primeiros anos de vida são essenciais para o desenvolvimento das potencialidades do indivíduo, sendo fundamental ao profissional ter conhecimento adequado e incentivar o estímulo do desenvolvimento neuropsicomotor, como também acompanhá-lo rotineiramente. Algumas crianças, mesmo sadias e bem nutridas, por não terem recebido estimulação em quantidade, qualidade e diversidade adequada, deixam de desenvolver plenamente suas potencialidades ${ }^{(18)}$. Portanto, faz-se necessário o acompanhamento cauteloso do desenvolvimento infantil no serviço de APS, sendo o enfermeiro um dos profissionais responsáveis por essa ação ${ }^{(19)}$.

A vigilância do desenvolvimento infantil em menores de dois anos caracteriza-se como uma estratégia capaz de atender às necessidades globais da criança, pois possibilita avaliar os marcos do desenvolvimento para faixa etária e os fatores de risco para atraso neste processo. Nesta, cabe à enfermeira considerar as informações fornecidas pelos pais acerca do comportamento de seu filho, informá-los sobre este processo na criança, orientá-los quanto à importância da estimulação adequada, visto que quanto mais precoce a detecção de déficit no desenvolvimento, maior as possibilidades para reversão do quadro ${ }^{(17)}$.

A fragilidade na prática educativa para a promoção do desenvolvimento é um achado preocupante frente à vigilância do desenvolvimento infantil, tendo em vista que no atendimento à criança, parece que as enfermeiras não estabelecem um diálogo que contemple esse aspecto, fornecendo apenas orientações para alguns cuidados práticos. Portanto, perdem a oportunidade de agir como facilitadores e promotores para o desenvolvimento saudável da criança, apesar desta ação ser inerente às competências profissionais para promoção da saúde infantil.

Estudo $^{(20)}$ de intervenção realizado em Taiwan objetivando capacitar famílias para a estimulação de crianças com risco para alterações no desenvolvimento, constatou melhora dessas crianças, confirmando a importância do preparo familiar para a promoção do desenvolvimento saudável.
Como uma das ações educativas mencionadas, a atividade de sala de espera é tida como um espaço dinâmico e rico que favorece trocas de experiências entre os usuários, consolidando seu potencial educativo. Ademais, gera maior acolhimento e humanização no cuidado aos usuários, efetivando a aproximação entre a comunidade e os serviços de saúde, e auxiliando na prevenção de doenças e na promoção da saúde da população(21). Logo, esse cenário deve ser aproveitado para realizar a promoção do desenvolvimento saudável das crianças por meio de atividades de educativas, proporcionando discussão sobre a temática e acompanhamento coletivo.

Nesse aspecto, estudo ${ }^{(22)}$ objetivando descrever o processo de implantação e desenvolvimento do acompanhamento coletivo do crescimento e desenvolvimento das crianças pela enfermagem, destacou o fazer coletivo como uma ferramenta de aprendizagem inovadora, válida, mas que enfrenta a dificuldade de romper com a prática individual comumente realizada que, por vezes, torna-se o fator principal que ocasiona alguns entraves na execução da ação.

Assim, a realização do processo educativo por meio de atividades grupais é um dos desafios enfrentados pelos profissionais da saúde na ESF, por propor novas situações de saúde para a comunidade, e necessitar da participação ativa de toda a equipe. Contudo, essas atividades coletivas merecem destaque por abordar a vigilância em saúde, envolvendo a promoção, a avaliação e a recuperação da saúde em todos os níveis de assistência, de maneira integrada e multiprofissional ${ }^{(23-24)}$.

Além das dificuldades vivenciadas pelas enfermeiras, decorrentes das condições de trabalho, é notório que algumas não estão preparadas para as atividades de educação em saúde, assim como para as ações voltadas para a vigilância do desenvolvimento infantil. Tal fato evidencia a necessidade da educação permanente, uma importante ferramenta que deve ser utilizada para atender às necessidades das equipes de saúde da família ${ }^{(13)}$.

\section{CONSIDERAÇÕES FINAIS}

Algumas enfermeiras apresentam visão coerente sobre a educação em saúde, conforme os princípios propostos pelo Ministério da Saúde. Porém, ainda é comum ver que outras referiram práticas educativas arraigadas no modelo centrado 
na doença. Vale ressaltar que há dualidade quanto à concepção de educação em saúde apresentadas nos discursos, uma vez que seus ideais são pautados tanto em modelos voltados para a promoção da saúde como no modelo de atenção médico centrado. Considerando a relevância do processo de vigilância em saúde no contexto da atenção à criança, é importante que as enfermeiras compreendam a dimensão de suas ações de educação em saúde como inerentes à prática de promoção da saúde no processo saúdedoença.

Nessa perspectiva, percebe-se nos relatos que é comum enfatizarem as orientações pertinentes à imunização, alimentação e higienização como ações de educação em saúde, no entanto, não remetem às práticas educativas desenvolvidas junto às famílias que promovam o desenvolvimento neuropsicomotor das crianças. Fato este inquietante, pois a vigilância do desenvolvimento infantil é uma atividade preconizada nas políticas públicas de saúde da criança, com vistas à promoção da saúde e prevenção de danos a essa população. Desta forma, é importante que os enfermeiros durante seus atendimentos à criança executem ações voltadas ao desenvolvimento neuropsicomotor, visando o estímulo e monitoramento da saúde infantil, compreendendo-os e reconhecendo-os, também, como parte integrante do processo de educativo.

Assim, este estudo possibilitou compreender que algumas enfermeiras ainda não conseguem articular educação em saúde às suas atividades de promoção do desenvolvimento infantil saudável. Tal fato talvez deva-se à fragilidade na formação, à concepção de saúde higienista e médico-centrada ainda presente, como também às demandas aumentadas nos serviços de saúde. Esta última exige, muitas vezes, mudanças no processo de trabalho do profissional, o qual passa a priorizar a quantidade em detrimento da qualidade, impossibilitando a realização das ações de educação em saúde por falta de tempo.

Nesse contexto, observa-se uma concepção simplificada do conceito de educação em saúde em relação à vigilância do desenvolvimento infantil. Fornecer orientações não é suficiente para promover uma melhora na qualidade de vida da população infantil. É preciso acompanhar efetivamente o crescimento e desenvolvimento infantil, e capacitar as mães para detectarem precocemente possíveis problemas que possam afetar o desenvolvimento neuropsicomotor de seus filhos e estimulá-los conforme sua faixa etária.

Ante o exposto, é notória a necessidade de sensibilização de enfermeiras para valorização de suas competências envolvidas na promoção do desenvolvimento saudável, especialmente na vigilância do crescimento e desenvolvimento infantil, a fim de adotar medidas para qualificar a atenção à criança na APS.

Este estudo apresenta limitações pelo fato de ter sido realizado por meio de entrevista gravada, podendo ter provocado alterações no comportamento dos participantes. Além disso, foi realizada com um grupo pequeno de profissionais, ressaltando-se a necessidade de que seja ampliado para todo o município pesquisado. Contudo, espera-se que ele contribua para uma reflexão acerca das atividades educativas voltadas ao desenvolvimento infantil saudável no âmbito da ESF, assim como possa instigar a realização de novas pesquisas na área.

\section{REFERÊNCIAS}

1. Silva DI, Chiesa AM, Veríssimo MLOR, Mazza VA. Vulnerabilidade da criança diante de situações adversas ao seu desenvolvimento: proposta de matriz analítica. Rev. esc. enferm. USP. 2013; 47(6):1397-1402.

2. Olusanya BO. Priorities for early childhood development in low-income countries. J Dev Behav Pediatr. 2011; 32(6):476-81.

3. Santos RV, Penna CMM. A educação em saúde como estratégia para o cuidado à gestante, puérpera e ao recém-nascido. Texto Contexto Enferm. 2009; 18(4):652-60.

4. Monteiro FPM, Araujo TL, Ximenes LB, Vieira NFC. Ações de promoção da saúde realizadas por enfermeiros na avaliação do crescimento e desenvolvimento infantil. Cienc. enferm. 2014; 20(1):97110 [acesso em 02 fev 2015]. Disponível: http://dx.doi. org/10.4067/S0717-95532014000100009.

5. Monteiro Al, Lima KYN, Santos ADB, Teixeira GB, Macêdo IP. Humanização do atendimento à criança na atenção básica: visão dos profissionais. Rev Rene. 2012; 13(4):724-33.

6. Barbosa LA, Sampaio ALA, Melo ALA, Macedo APN, Machado MFAS. Educação em saúde como instrumento na prevenção de parasitoses. RBPS. 2009; 22(4):272-8.

7. Reichert APS, Almeida AB, Souza LC, Silva MEA, Collet N. Vigilância do crescimento infantil: conhecimento e práticas de enfermeiros da atenção primária à saúde. Rev Rene. 2012; 13(1):114-26. 
8. Minayo MCS. O desafio do conhecimento: pesquisa qualitativa em saúde. 11ª ed. São Paulo: Hucitec; 2012.

9. Vieira VCL, Fernandes CA, Demitto MO, Bercini LO, Scochi MJ, Marcon SS. Puericultura na atenção primária à saúde: atuação do enfermeiro. Cogitare Enferm. 2012; 17(1):119-25.

10. Pereira MM, Penha TP, Vaz EMC, Collet N, Reichert APS. Concepções e práticas dos profissionais da estratégia saúde da família sobre educação em saúde. Texto Contexto Enferm. 2014; 23(1):167-75.

11. Tesser CD, Garcia AV, Vendruscolo C, Argenta CE. Estratégia saúde da família e análise da realidade social: subsídios para políticas de promoção da saúde e educação permanente. Ciênc. saúde coletiva. 2011; 16(11):4295-306 [acesso em 10 mai 2013]. Disponível: http://dx.doi.org/10.1590/S1413-81232011001200002.

12. Beserra EP, Alves MDS, Pinheiro PNC, Vieira NFC. Educação ambiental e enfermagem: uma integração necessária. Rev. bras. enferm. 2010; 63(5):848-52.

13. Rodrigues CC, Ribeiro KSQS. Promoção da saúde: a concepção dos profissionais de uma unidade de saúde da família. Trab. educ. saúde. 2012; 10(2):235-55.

14. Rodrigues D, Santos VE. A Educação em Saúde na Estratégia Saúde da Família: uma revisão bibliográfica das publicações científicas no Brasil. J. Health Sci Inst. 2010; 28(4):321-4.

15. Heidemann ITSB, Wosny AM, Boehs AE. Promoção da Saúde na Atenção Básica: estudo baseado no método de Paulo Freire. Ciênc. saúde coletiva. 2014; 19(8):3553-9.

16. David HMSL, Acioli S. Mudanças na formação e no trabalho de enfermagem: uma perspectiva da educação popular e de saúde. Rev. bras. enferm. 2010; 63(1) [acesso em 23 mai 2013]. Disponível: http://dx.doi. org/10.1590/S0034-71672010000100021.

17. Reichert APS, Vasconcelos MGL, Eickmann SH, Lima MC. Avaliação da implementação de uma intervenção educativa em vigilância do desenvolvimento infantil com enfermeiros. Rev. esc. enferm. USP. 2012; 46(5):1049-56.

18. Oliveira DKS, Nascimento DDG, Marcolino FF. Perceptions of family caregivers and professionals in the family health strategy related to the care and neuropsychomotor development of children. J. Hum. Growth Dev. 2012; 22(2):142-50.

19. Oliveira LL, Costa VMR, Requeijo MR, Rebolledo RS, Pimenta AF, Lemos SMA. Desenvolvimento infantil: concordância entre a caderneta de saúde da criança e o manual para vigilância do desenvolvimento infantil. Rev. paul. pediatr. 2012; 30(4):479-85.
20. Hwang AW, Chao MY, Liu SW. A randomized controlled trial of routines-based early intervention for children with or at risk for developmental delay. Res. Dev. Disabil. 2013; 34(10):3112-23.

21. Rodrigues AD, Dallanora CR, Rosa J, Germani ARM. Sala de espera: um ambiente para efetivar a educação em saúde. Vivências. 2009; 5(7):101-06.

22. Alves GG, Aerts D. As práticas educativas em saúde e a Estratégia Saúde da Família. Ciênc. saúde coletiva. 2011; 16(1):319-25.

23. Santos NO, Santos VCF, Santos MP, Roese A. (Des)Encontros: A educação em saúde na formação profissional do enfermeiro. Rev. Enferm. UFPE online. 2013; 7(n.esp) [acesso em 14 jul 2013]. Disponível: http://www.revista.ufpe.br/revistaenfermagem/index. php/revista/article/view/3198.

24. Monteiro AI, Macedo IP, Santos ADB, Araújo WM. A enfermagem e o fazer coletivo: acompanhando o crescimento e o desenvolvimento da criança. Rev Rene. 2011; 12(1):73-80. 